\title{
Analyzing Some Behavior of a Beam with Different Crack Positions Transversely inside It
}

\author{
Behrooz Yazdizadeh \\ Department of Mechanics, Kerman Branch, Islamic Azad University, Kerman, Iran \\ Email: behyazd@iran.ir
}

Received October 4, 2012; revised November 6, 2012; accepted November 25, 2012

\begin{abstract}
Vertical displacement, critical Euler buckling load and vibration behavior of a cracked beam are considered in this research. The crack inside the beam is placed in different positions and results compared for each crack position. On first Eigenvalue of free vibration results, there is a border that first Eigenvalue of free vibration does not change if center of crack is located on that border, and after that border, the first Eigenvalue of free vibration is increased that is a counterexample relation of critical Euler buckling load and first Eigenvalue of free vibration.
\end{abstract}

Keywords: Crack; Mesh; Vibration; Euler Buckling; Displacement

\section{Introduction}

Fracture mechanics, was originated by Wieghardt and Inglis [1]. Both independently showed that cavities and flaws in continuum materials act as stress concentrators which, in the limit of sharp edges (cracks), produce infinite stress at the tip [2]. A fairly thorough description of the approaches for solving the crack problems is made by many researchers [3-6]. These were the first attempts to bring closer the theories of fracture mechanics (FM) and continuum mechanics (CM). About the same time, the Finite Element Method (FEM) and digital computers dashed into the engineering community as a gifted means for quantifying solutions in structural and solid mechanics. Naturally, fracture mechanic researchers implemented their FE methods, while continuum mechanic researchers implemented theirs [7].

Over the years, the finite element technique has been so well established that today it is considered one of the best methods for solving a wide variety of practical problems efficiently and is more and more feasible, provided that finite element (FE) models can be shown to be correct [8]. It can be used for the solution of any problem simply by changing the input data [9].

A lot of effort has been spent in the last 30 years to investigate and treatment the observed drawbacks of (FE) method [10] and Finite element analysis is one of the usual ways to solve crack problems.

In this research, some beam characteristic behavior comparing with each other in different crack Positions inside the beam. These comparing consist of maximum vertical displacement under simple bending, first mode first Eigenvalue of free vibration and critical Euler buckling load.

Critical Euler buckling load for single beam-columns can be evaluated from analytical expressions. These solutions, as given for various boundary conditions, follow the first analytical method given by Euler [11] for predicting the reduced strengths of slender columns. The finite elements method can be easily implemented for beam elements without cracks since the stiffness and generalized geometrical stiffness matrices of a noncracked beam are already commonly known (for example in [12]). However, the situation essentially changes if the structural elements are transversely cracked. Two dimension (2D) or three dimension (3D) finite element analyzes must be implemented in order to achieve a complete model of the structure. When studying the elastic Euler buckling load of a column, it is necessary to determine the maximum load at which the structure remains in equilibrium at the deformed position.

Stress intensity factor, normal, and shear stresses (at the tip of the crack) are calculated for simple cracked beam under vertical pressure before [13].

Here we examine changes of some parameters of a beam with different crack position inside of it for conclusion that whether crack position has an important role to change the vertical displacement, first Eigenvalue of free vibration and critical Euler buckling load or not.

\section{Material and Methods}

Consider a beam as shown in Figure 1. This beam loaded with a vertical load at end of it then calculating 


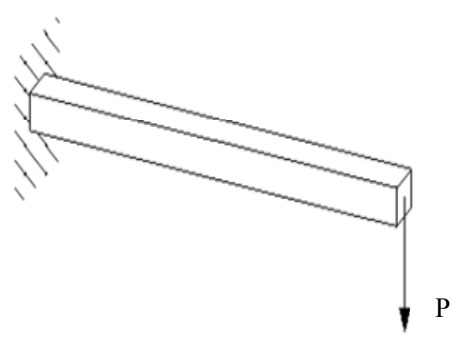

Figure 1. Beam under loading.

the Maximum vertical displacement at the end of it. These procedures do again with applying a horizontal crack inside the beam as shown in Figure 2. Also again when crack moves along the beam vertically and horizontally. They are fifteen Positions that consider for crack. For each position, vertical displacement, first Eigenvalue of free vibration and critical Euler buckling load are determined and compared with each other.

The beam has 2 meter $(\mathrm{m})$ length and $0.12 \mathrm{~m}$ width with thickness of $0.12 \mathrm{~m}$. (Young's modulus) $E=2 \times$ $10^{12} \mathrm{~Pa}$, (Poisson's ratio $v=0$, (density) $D=7800 \mathrm{~kg} / \mathrm{m}^{3}$ and (load at the end of the beam) $P=10 \mathrm{kN}$.

\section{Crack parameters are as follows:}

Length $=0.38$ meter;

Depth $=$ through the beam;

Space between surface $=0.002$ meter;

Radius of crack at tip $=0.002$ meter.

\section{Center of crack locations are as follows:}

Horizontally ( $x$ dimensions - fixed end of the beam is the origin of axes):

1) $0.2 \mathrm{~m}$; 2) $0.6 \mathrm{~m}$; 3) $1 \mathrm{~m}$; 4) $1.4 \mathrm{~m}$; 5) $1.8 \mathrm{~m}$.

Vertically (y dimensions - lower surface of the beam is the origin of axes):

1) $0.02 \mathrm{~m}$; 2) $0.06 \mathrm{~m}$; 3) $0.1 \mathrm{~m}$.

The displacement formula for a beam [14]:

$$
E I \frac{\partial^{4} y}{\partial x^{4}}=0
$$

where $E$ is module of elasticity; $I$ is moment of inertia; $y$ is vertical displacement of a point in $x$ position.

The solution of above differential equation with boundary conditions is as follows (See Figure 1):

$$
y=\frac{p}{6 E I}\left(2 L^{3}-3 L^{2} x+x^{3}\right)
$$

The maximum displacement (at $x=0)$ is $\left(\frac{P L^{3}}{3 E I}\right)$.

There are several ways to calculate the beam frequency. Relation (2) is obtained from Rayleigh approximation method and the error of this approximated solution is less than $0.5 \%$ [15].

$$
f=\frac{1}{2 \pi} k \times \sqrt{\frac{E I g}{\omega L^{4}}}
$$

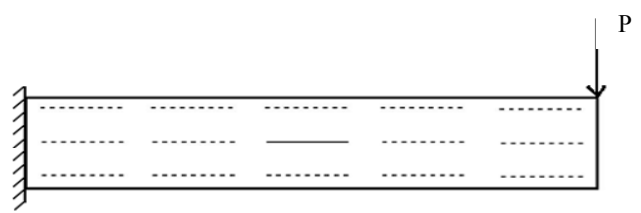

Figure 2. Crack positions inside the beam.

where $f$ is first mode lateral frequency; $\omega$ is weight per unit length; $g$ is the volume coefficient that is chooses here to be equal to 1 and $k$ is the constant that is equal to 3.53 .

The critical first Eigenvalue-Euler buckling load $\left(P_{c r}\right)$ of a beam is determined from Equation (3) [14]:

$$
P_{c r}=\frac{\pi^{2} E I}{L^{2}}
$$

where $E$ is module of elasticity; $I$ is moment of inertia and $L$ is the length of a beam.

If there is no crack inside the beam then from relations (1)-(3), maximum displacement vertical displacement, vibration and first Eigenvalue-Euler buckling load of the beam can obtained that they are $7.72 \times 10^{-4} \mathrm{~m}, 77.91 \mathrm{~s}^{-1}$, $21.30 \times 10^{3} \mathrm{kN}$, respectively.

For every location maximum vertical displacement, first mode vibration and first critical Euler Euler buckling load are obtained.

To reduce the error of meshing (because for each new crack location we must mesh the beam again, which is different from previous one), whole shape of the beam does not mesh at once. The beam divided into 15 equivalent parts that are shown in Figure 3. One part is for crack element and another are normal beam parts. Hence, a part of the beam, which includes crack, can be moved to different location. Other divided parts of the beam could be moved to different location too. In this condition, meshing of the whole area of the beam is constant for every crack position.

Note that in ANSYS software we cannot model the crack with two crack tips. Only one tip is allowable. To solve this problem, half of the crack is modeling and meshing (as shown in Figure 4) then with mirror option in ANSYS software, copy it to another part of the crack (see Figures 5 and 6).

After each modeling, all nodes and elements on the borders (edge) of each part must be merging to each other. Then construction of the whole beam is complete.

After modeling of each crack position and apply boundary conditions, maximum vertical displacement, frequency and Euler Euler buckling of the beam are calculated and analyzed.

Now we consider the results of this problem.

\section{Results and Discussion}

Tables 1-3 show maximum vertical displacement; first 


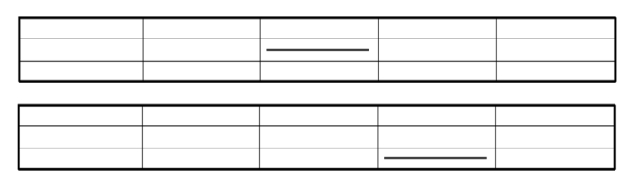

Figure 3. Beam divided to 15 parts (each part meshes separately).

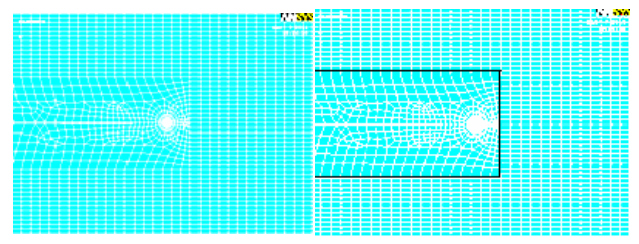

Figure 4. Half of crack modeling in ANSYS software (crack part of the beam is specified in right).

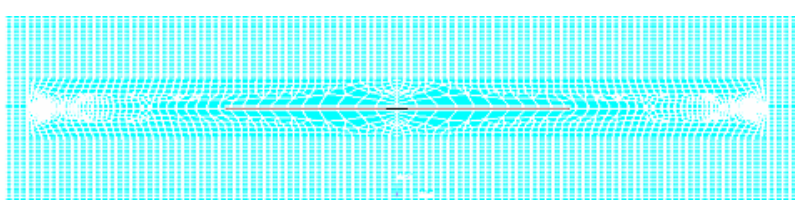

Figure 5. Total crack modeling in ANSYS software.

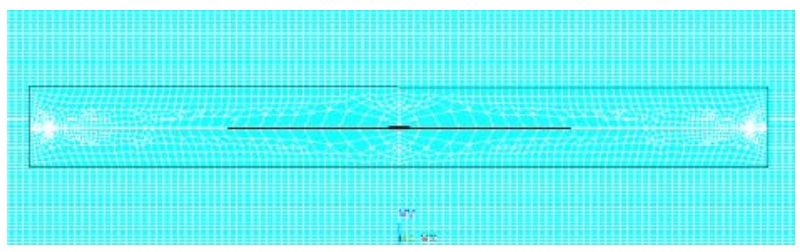

Figure 6. Total crack modeling in ANSYS software (crack part of the beam is bounded).

Table 1. Vertical maximum displacement data (m).

\begin{tabular}{ccccccc}
\hline \multirow{2}{*}{$\begin{array}{c}\text { Simple beam } \\
\text { (without crack) }\end{array}$} & \multirow{2}{*}{$\begin{array}{c}\text { Center of crack } \\
\text { positions vertically }\end{array}$} & \multicolumn{5}{c}{ Center of crack positions horizontally } \\
\cline { 3 - 7 } & 0.1 & 0.2 & 0.6 & 1 & 1.4 & 1.8 \\
\hline \multirow{2}{*}{$7.73 \times 10^{-3}$} & 0.06 & $7.79 \times 10^{-3}$ & $7.77 \times 10^{-3}$ & $7.76 \times 10^{-3}$ & $7.75 \times 10^{-3}$ & $7.75 \times 10^{-3}$ \\
& 0.02 & $7.79 \times 10^{-3}$ & $7.77 \times 10^{-3}$ & $7.76 \times 10^{-3}$ & $7.75 \times 10^{-3}$ & $7.75 \times 10^{-3}$ \\
\hline
\end{tabular}

Table 2. First mode first Eigenvalue of free vibration data (1/s).

\begin{tabular}{ccccccc}
\hline \multirow{2}{*}{$\begin{array}{c}\text { Simple beam } \\
\text { (without crack) }\end{array}$} & $\begin{array}{c}\text { Center of crack positions } \\
\text { vertically }\end{array}$ & \multicolumn{5}{c}{ Center of crack positions horizontally } \\
\cline { 3 - 7 } & 0.1 & 77.013 & 77.153 & 1 & 1.4 & 1.8 \\
\hline \multirow{3}{*}{77.418} & 0.06 & 76.833 & 76.86 & 77.015 & 77.29 & 77.583 \\
& 0.02 & 77.013 & 77.153 & 77.29 & 77.435 & 77.564 \\
\hline
\end{tabular}

Table 3. First Euler buckling critical load data (N).

\begin{tabular}{ccccccc}
\hline \multirow{2}{*}{$\begin{array}{c}\text { Simple beam } \\
\text { (without crack) }\end{array}$} & $\begin{array}{c}\text { Center of crack } \\
\text { positions vertically }\end{array}$ & \multicolumn{5}{c}{ Center of crack positions horizontally } \\
\cline { 3 - 7 } & 0.1 & 0.2 & 0.6 & 1 & 1.4 & 1.8 \\
\hline \multirow{2}{*}{$2.13 \times 10^{7}$} & 0.06 & $2.12 \times 10^{7}$ & $2.12 \times 10^{7}$ & $2.12 \times 10^{7}$ & $2.12 \times 10^{7}$ & $2.12 \times 10^{7}$ \\
& 0.02 & $2.13 \times 10^{7}$ & $2.12 \times 10^{7}$ & $2.12 \times 10^{7}$ & $2.11 \times 10^{7}$ & $2.10 \times 10^{7}$ \\
& $2.12 \times 10^{7}$ & $2.12 \times 10^{7}$ & $2.12 \times 10^{7}$ & $2.12 \times 10^{3}$ & $2.12 \times 10^{7}$ \\
\hline
\end{tabular}

mode vibration and first critical Euler buckling load respectively and Figures 7-10 are showing these data on diagrams (graphs).

Note that Figures $\mathbf{7}$ and $\mathbf{8}$ are equivalent. For having the best view of diagrams, (2D) view is chosen instead of (3D) view.

In Figure 8, when crack is not placed at the center and moved horizontally from fixed point toward the end of the beam displacement decreased. However, when crack is placed at the center, there is some little increasing of displacement.

In Figure 9, when crack moved horizontally from fixed point toward the end of the beam first Eigenvalue of free vibration increased up to near the first Eigenvalue of free vibration of the beam without crack. When crack moved vertically first Eigenvalue of free vibration increased. It means that when there is a crack near the edges of a beam, first Eigenvalue of free vibration is more than when crack is located in the middle of the beam.

In Figure 10, when crack position, is at the center and moved horizontally from fixed point toward the end of the beam critical load reduced, meaning that the system is more unstable. 


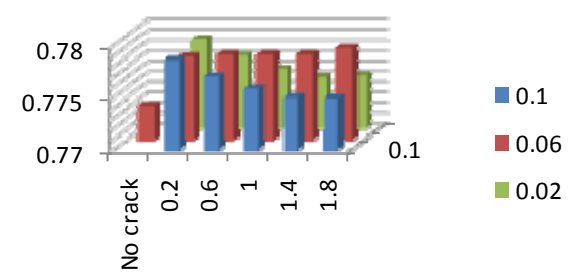

Figure 7. Vertical maximum displacement diagram (3D view) $\left(\times 10^{-3} \mathrm{~m}\right)$.

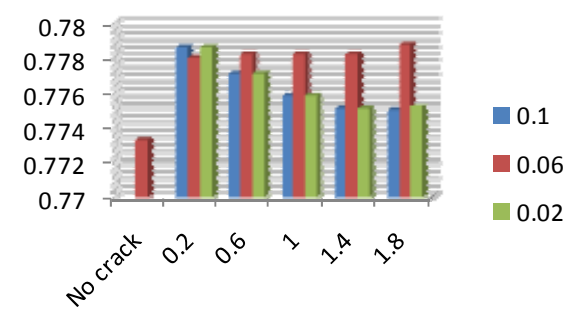

Figure 8. Vertical maximum displacement diagram (2D view) $\left(\times 10^{-3} \mathrm{~m}\right)$.

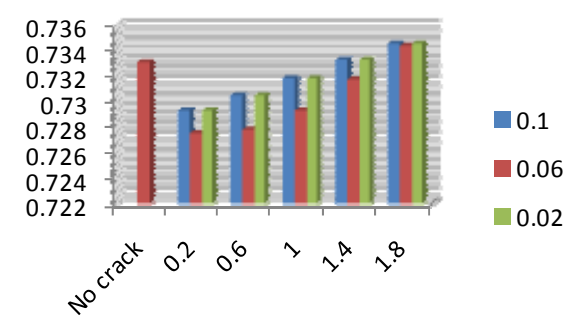

Figure 9. First mode first Eigenvalue of free vibration diagram (2D view).

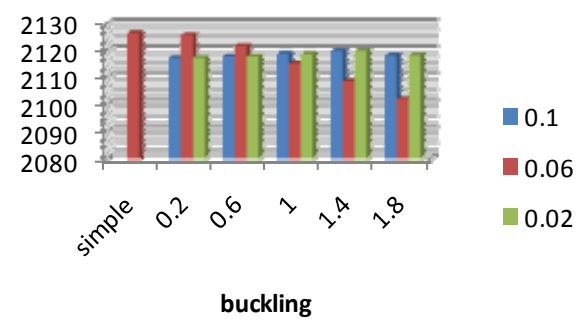

Figure 10. First buckling critical load diagram (2D view) $\left(\times 10^{4} \mathrm{~N}\right)$.

There is more stability for beam when crack not placed at the center of the beam except near the fixed point (left of the beam). In this chart, we show only (2D) display instead of (3D) chart for better comparing. In addition, when crack is placed at the center and moved horizontally from fixed point toward the end of the beam first Euler buckling critical load is decreased. However, when crack is not placed at the center, there is some little increasing of first Euler buckling critical load from fixed point toward the end of the beam.

In general discussion; different shape of characteristic behavior of the beam when crack positioned at the center may be for that the center is located in the neutral line of

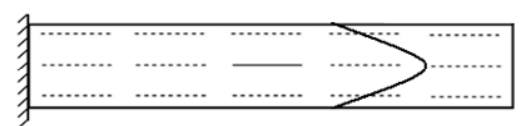

Figure 11. First Eigenvalue of free vibration border for the beam.

the beam that there is no any tensile stress on it or displacement.

Percentage different of Maximum value of crack position with a non-crack beam shows the different below $5 \%$ that means insignificant distinct for all condition that discussed:

Euler buckling different: $1.14 \%$;

First Eigenvalue of free vibration different: $0.754 \%$;

Displacement different: $0.712 \%$.

\section{Conclusions}

Results show that vertical displacement increases when crack is located in the center of the beam (respect to $y$-axes) and critical load Euler buckling is decreasing in that position (when crack is located in the center). However, in first Eigenvalue of free vibration when the crack is moved near the beam end, first Eigenvalue of free vibration increased more than the beam first Eigenvalue of free vibration when there is no crack inside it. It can be conclude that on first Eigenvalue of free vibration results, there is a border that first Eigenvalue of free vibration does not change if one crack is located on that border, and after that border, the first Eigenvalue of free vibration is increased. The border is shown in Figure 11.

Vertical displacement is increased or constant when crack is moved from the fixed pint to free end of the beam (left to right) when crack is located near the edge of the beam but when crack is located in the center of the beam height and again crack is moved from the fixed pint to free end of the beam vertical displacement is decrease.

First Euler buckling critical load diagram shows a same result as first Eigenvalue of free vibration, except that for all crack location critical loads are less that the beam without cracks, however first Eigenvalue of free vibration is increased (after the border that shows in Figure 11) more than that one from the beam without cracks.

Finally the border in the beam shows that after that the first Eigenvalue of free vibration is increased more than a simple beam without crack but critical Euler buckling load is decreased lower that a simple beam without crack that is a counterexample relation of critical Euler buckling load and first Eigenvalue of free vibration.

\section{REFERENCES}

[1] C. E. Inglis, "Stresses in a Plate Due to the Presence of 
Cracks and Sharp Corners," SPIE Milestone Series, Vol. 137, 1913, pp. 3-17.

[2] A. Caballero and A. Dyskin, "Mesh Scalability in Direct Finite Element Simulation of Brittle Fracture," Engineering Fracture Mechanics, Vol. 75, No. 14, 2008, pp. 40664084. doi:10.1016/j.engfracmech.2008.03.007

[3] F. Erdogan, "Stress Intensity Factors," Journal of Applied Mechanics, Vol. 50, No. 4b, 1983, pp. 992-1002. doi:10.1115/1.3167212

[4] H. Liebowitz and G. C. Sih, "Mathematical Theories of Brittle Fracture," Academic Press, Waltham, 1968.

[5] M. P. Savruk, "Two-Dimensional Elasticity Problems for Bodies with Cracks," Savruk, Kiev, 1981.

[6] I. N. Sneddon and M. Lowengrub, "Crack Problems in the Classical Theory of Elasticity," Wiley, Hoboken, 1969.

[7] M. Cervera and M. Chiumenti, "Mesh Objective Tensile Cracking via a Local Continuum Damage Model and a Crack Tracking Technique," Computer Methods in Applied Mechanics and Engineering, Vol. 196, No. 1-3, 2006, pp. 304-320. doi:10.1016/j.cma.2006.04.008

[8] K. K. Gupta and J. L. Meek, "A Brief History of the Beginning of the Finite Element Method," International
Journal for Numerical Methods in Engineering, Vol. 39, No. 22, 1996, pp 3761-3774. doi:10.1002/(SICI)1097-0207(19961130)39:22<3761::AI D-NME22>3.3.CO;2-X

[9] S. S. Rao, "The Finite Element Method in Engineering," 4th Edition, Butterworth-Heinemann, Oxford, 2011.

[10] M. Cervera, "An Orthotropic Mesh Corrected Crack Model," Computer Methods in Applied Mechanics and Engineering, Vol. 197, No. 17-18, 2008, pp. 1603-1619. doi:10.1016/j.cma.2007.12.007

[11] L. Euler, "On the Strength of Columns," American Journal of Physics, Vol. 15, No. 4, 1947, p. 315. doi:10.1119/1.1990953

[12] P. Bhatt and W. T. Marshall, "Structures," 4th Edition, Longman, Harlow, 1999.

[13] B. Yazdizadeh, "Analyzing Some Behavior of a Cracked Beam under Pressure," International Journal of Science and Technology, Vol. 2, No. 9, 2012, pp. 681-688.

[14] E. P. Popov and T. A. Balan, "Engineering Mechanics of Solids," 2nd Edition, Prentice-Hall, Upper Saddle River, 1998.

[15] S. Timoshenko, "Vibration Problems in Engineering," 4th Edition, Wolfenden Press, New York, 1974. 